\title{
Searching for galaxy clusters in the Kilo-Degree Survey ${ }^{\star}$
}

\author{
M. Radovich ${ }^{1}$, E. Puddu ${ }^{2}$, F. Bellagamba ${ }^{3,4}$, M. Roncarelli ${ }^{3,4}$, L. Moscardini ${ }^{3,4,5}$, S. Bardelli ${ }^{4}$, A. Grado ${ }^{2}$, F. Getman ${ }^{2}$, \\ M. Maturi ${ }^{6}$, Z. Huang ${ }^{2}$, N. Napolitano ${ }^{2}$, J. McFarland ${ }^{7}$, E. Valentijn ${ }^{7}$, and M. Bilicki ${ }^{8}$
}

\author{
1 INAF-Osservatorio Astronomico di Padova, vicolo dell'Osservatorio 5, 35122 Padova, Italy \\ e-mail: mario.radovich@oapd.inaf.it \\ 2 INAF-Osservatorio Astronomico di Capodimonte, Salita Moiariello 16, 80131 Napoli, Italy \\ 3 Dipartimento di Fisica e Astronomia, Alma Mater Studiorum - Università di Bologna, viale Berti Pichat 6/2, 40127 Bologna, Italy \\ 4 INAF-Osservatorio Astronomico di Bologna, via Ranzani 1, 40127 Bologna, Italy \\ 5 INFN - Sezione di Bologna, viale Berti-Pichat 6/2, 40127 Bologna, Italy \\ 6 Zentrum für Astronomie, Universitatät Heidelberg, Philosophenweg 12, 69120 Heidelberg, Germany \\ 7 Kapteyn Astronomical Institute, PO Box 800, 9700 AV Groningen, The Netherlands \\ ${ }^{8}$ Leiden Observatory, Leiden University, PO Box 9513, 2300 RA Leiden, The Netherlands
}

Received 20 July 2016 / Accepted 23 December 2016

\begin{abstract}
Aims. In this paper, we present the tools used to search for galaxy clusters in the Kilo Degree Survey (KiDS), and our first results. Methods. The cluster detection is based on an implementation of the optimal filtering technique that enables us to identify clusters as over-densities in the distribution of galaxies using their positions on the sky, magnitudes, and photometric redshifts. The contamination and completeness of the cluster catalog are derived using mock catalogs based on the data themselves. The optimal signal to noise threshold for the cluster detection is obtained by randomizing the galaxy positions and selecting the value that produces a contamination of less than $20 \%$. Starting from a subset of clusters detected with high significance at low redshifts, we shift them to higher redshifts to estimate the completeness as a function of redshift: the average completeness is $\sim 85 \%$. An estimate of the mass of the clusters is derived using the richness as a proxy.

Results. We obtained 1858 candidate clusters with redshift $0<z_{c}<0.7$ and mass $10^{13.5}<M_{500}<10^{15} M_{\odot}$ in an area of 114 sq. degrees (KiDS ESO-DR2). A comparison with publicly available Sloan Digital Sky Survey (SDSS)-based cluster catalogs shows that we match more than $50 \%$ of the clusters $(77 \%$ in the case of the redMaPPer catalog). We also cross-matched our cluster catalog with the Abell clusters, and clusters found by XMM and in the Planck-SZ survey; however, only a small number of them lie inside the KiDS area currently available.
\end{abstract}

Key words. galaxies: clusters: general - galaxies: distances and redshifts

\section{Introduction}

Clusters of galaxies are described as the most massive collapsed structures in the Universe. They represent a powerful tool for cosmological studies (Allen et al. 2011), making it possible to probe the formation history of cosmic structures at different redshifts, and to constrain the measurement of cosmological parameters, such as the matter density parameter $\Omega_{\mathrm{M}}$ and the power spectrum normalization $\sigma_{8}$ (see e.g. White et al. 1993; Eke et al. 1998; Sartoris et al. 2016, and references). In this perspective, it becomes crucial that photometric surveys be able to supply a statistically significant sample for the detection of clusters over large sky areas, compared to X-rays surveys, for example, that cover smaller patches. Among the available surveys of different sizes and depths, one of the landmarks is the Sloan Digital Sky Survey (SDSS; York et al. 2000), probing the low-redshift universe with an imaging sky coverage of 14555 sq. degrees. Ongoing programs, like the Kilo Degree Survey $^{1}$ (KiDS, de Jong et al. 2013) and the Dark Energy Survey (DES, The Dark Energy Survey Collaboration 2005), will

\footnotetext{
$\star$ The catalog is available at http://kids.strw. leidenuniv.nl/DR2 and at the CDS

via anonymous ftp to cdsarc.u-strasbg. fr (130.79.128.5) or via http://cdsarc.u-strasbg.fr/viz-bin/qcat?]/A+A/598/A107 1 http://kids.strw.leidenuniv.nl
}

provide samples of clusters spanning a wider range of redshift and mass, thanks to their superior depth. When completed, KiDS will cover 1500 sq. degrees in the ugri bands, with optimal seeing conditions in the $r$-band $\left(<0.8^{\prime \prime}\right)$; DES will cover a larger area (5000 sq. degrees in griZY), with an image quality between SDSS and KiDS, and typical seeing $\sim 1^{\prime \prime}$ (Melchior et al. 2015). Both surveys will make it possible to extend the search of galaxy clusters to redshifts $z \sim 0.9$ (see Rykoff et al. 2016, for results based on the DES Science Verification data). Finally, the European Space Agency Cosmic Vision mission Euclid (Laureijs et al. 2011), planned for launch in 2020, will be able to detect galaxy clusters up to redshift $z=2$ and to calibrate the cluster mass proxy with an accuracy $<10,30 \%$, using weak lensing and spectroscopic data, respectively (Sartoris et al. 2016).

In this paper we discuss the first results of the galaxy cluster search in the KiDS survey, based on the KiDS ESO-DR2 data release (KDR2; de Jong et al. 2015); improvements due to the availability of larger areas will be discussed in following papers. The cluster search method is based on Bellagamba et al. (2011, B11 hereafter). Compared to other methods employing the identification of the red sequence, for example, this approach presents the advantage that it does not search for a specific feature (e.g., color and brightness) of the cluster member galaxies. Instead, it enables us to simultaneously use the available information on the spatial distribution, magnitudes and 
photometric redshifts of the galaxies to find over-densities related to galaxy clusters. In addition to cluster searches using only optical data, this approach has been also used in cluster identifications based on X-ray (e.g. Pace et al. 2008; Tarrío et al. 2016) or weak lensing (Maturi et al. 2005) data.

The paper is organized as follows: the main features of KiDS and its data products are summarized in Sect. 2; details of the cluster finder algorithm are discussed in Sect. 3; the contamination and completeness of the cluster catalog are discussed in Sect. 4; richness and mass of the clusters are derived as explained in Sect. 5; Sect. 6 shows the properties of the cluster catalog by comparing it with other cluster datasets based on the SDSS Section 7 presents a summary of the clusters in the Abell, XMM, and Planck-SZ catalogs, which are located in the KDR2 area (see also Appendix A). Conclusions are given in Sect. 8.

\section{The Kilo-Degree Survey}

The Kilo Degree Survey is one of the ESO public surveys being performed with the OmegaCam wide-field camera (1 square degree field of view) mounted at the VLT Survey Telescope (VST). KiDS is designed to observe an area of 1500 sq. degrees in the ugri bands, with limiting $\mathrm{AB}$ magnitudes at $5 \sigma$ in a $2^{\prime \prime}$ aperture of respectively 24.3, 25.1, 24.9 and $23.8 \mathrm{mag}$ (KDR2). KiDS is made of two patches, one in the equatorial sky (KiDS-N) and the other around the south Galactic pole (KiDS-S).

The data processing and catalog extraction are done by the KiDS consortium using the AstroWISE system (Verdoes Kleijn et al. 2011). An extensive discussion of the survey and reduction techniques are given in KDR2. The data products included in the public ESO release are, for each band, the final stacked images, weight maps, and masks flagging regions with known problems (e.g., halos and spikes around bright stars, bad columns, etc.). Catalogs giving source positions and the photometry measured with SEXTRACTOR are derived both for each band independently and using the $r$-band as detection image. The star/galaxy classification is based on the CLASS_STAR parameter of SEXTRACTOR measured on $r$-band images, following the procedure described in KDR2, Sect. 4.5.1.

Photometric redshifts based on ugri photometry are also available within the KiDS collaboration: they were derived using both template fitting (Kuijken et al. 2015) with the BPZ code (Benítez 2000), and a machine-learning approach (Cavuoti et al. 2015) based on the MLPQNA method. The BPZ also provides the full redshift probability distribution function (PDF) that is required in our analysis to properly weight the contribution of galaxies. A discussion on the accuracy of the galaxy redshift distribution produced by BPZ and possible improvements are given by Choi et al. (2016), Hildebrandt et al. (2017).

The machine-learning approach provides very accurate photometric redshifts $(1 \sigma$ uncertainty in $\Delta z /(1+z)<0.03)$, which are less sensitive to uncertainties in photometric zero points, for example. However, machine-learning photometric redshifts are reliable only in the same parameter space sampled by the spectroscopic training sample, which was based on the SDSS in Cavuoti et al. (2015), and they do not provide the redshift PDF. Work is in progress to address these issues, using a deeper spectroscopic training sample, and developing a novel approach to derive PDFs for machine-learning photometric redshifts (Cavuoti et al. 2016). For this reason, in this analysis we opted for the template fitting photometric redshifts. We refer to Kuijken et al. (2015) for details on how they were derived: as displayed in their Fig. 12, for $z<0.7$ the rms scatter in $\Delta z /(1+z)$ is $<0.05$, and the outlier fraction is $<10 \%$. At the time the current analysis was done, they were computed only in the KIDS-N tiles ( 114 sq. degrees) overlapping with the Galaxy and Mass Assembly (GAMA) Survey (Driver et al. 2011).

The tiles available in KDR2 do not cover a contiguous area: for this reason, in this work we analyze each tile independently. Figure 1 shows the position on the sky of the tiles used for this paper. The latest KiDS public release, KiDS ESO-DR3, comprises an area of 440 sq. degrees (Hildebrandt et al. 2017): the extension of our analysis to the new data, including KIDS-S, is in progress and will be presented in a future paper.

The analysis in this work is based on the sources classified as galaxies in the KiDS catalogs, with an $r$-band magnitude brighter than the limiting magnitude at $10 \sigma, m_{10 \sigma} \sim 24.2$ mag. We removed from the catalogs all sources that were detected on spikes and halos nearby bright stars, where the density of spurious detections is higher and would increase the probability of obtaining false positive cluster candidates. To this end, we removed all detections where one of the following masking flags (see Table 4 in KDR2) is set to: 1 (readout spike), 2 (saturation core), 4 (diffraction spike), 16 (secondary halo), or 64 (bad pixels). Flagged regions were taken into account in the effective area computation for each KiDS tile.

An initial estimate of the number of expected clusters in KiDS vs. redshift was derived in KDR2 using the mock catalogs by Henriques et al. (2012) from the Millennium Simulation (Springel et al. 2005). According to this simulation, we would expect to detect $\sim 1980$ clusters with redshift $0<z<$ 0.7 and $13.5<\log \left(M / M_{\odot}\right)<15$ in an area of 114 sq. degrees ( 25900 clusters in the final KiDS area of 1500 sq. deg.).

\section{The cluster finding algorithm}

The search for regions with galaxy over-densities tracing clusters was performed using the Optimal Filtering technique, described in B11. A detailed description of the implementation of the algorithm is provided in a separate paper (Bellagamba et al., in prep.). The main idea of this approach is to describe the data in each point of the space as a sum of a cluster component $M$ and a field component $N$, which acts as noise for the cluster detection. Then, the amplitude $A$ of the cluster component at the point $\boldsymbol{x}_{\mathrm{c}}$ can be optimally estimated from the data $D$ via

$A\left(\boldsymbol{x}_{\mathrm{c}}\right)=\alpha^{-1} \int \frac{M\left(\boldsymbol{x}-\boldsymbol{x}_{\mathrm{c}}\right)}{N(\boldsymbol{x})}(D(\boldsymbol{x})-N(\boldsymbol{x})) \mathrm{d}^{n} x$,

where $\alpha$ is a normalisation constant defined as

$\alpha=\int \frac{M^{2}\left(\boldsymbol{x}-\boldsymbol{x}_{\mathrm{c}}\right)}{N(\boldsymbol{x})} \mathrm{d}^{n} x$.

Applying Eq. (1) means filtering the data $D$ with a kernel proportional to $M / N$. In our case, the data are: galaxy positions on the sky, magnitudes in the $r$ band, and photometric redshifts. Thus, we can make the first term of Eq. (1) more explicit as

$A\left(\boldsymbol{\theta}_{\mathrm{c}}, z_{\mathrm{c}}\right) \propto \sum_{i=1}^{N_{\mathrm{gal}}} \frac{M\left(\boldsymbol{\theta}_{i}-\boldsymbol{\theta}_{\mathrm{c}}, m_{i}\right) p_{i}\left(z_{\mathrm{c}}\right)}{N\left(m_{i}, z_{\mathrm{c}}\right)}$

where $\boldsymbol{\theta}_{\mathrm{c}}$ and $\boldsymbol{\theta}_{i}$ are the positions on the sky of the cluster center and of the $i$ th galaxy, respectively, $z_{\mathrm{c}}$ is the redshift of the cluster, and each galaxy is weighted by its own redshift probability distribution $p_{i}(z)$. The sum runs virtually over all the $N_{\text {gal }}$ galaxies of the catalog. By construction, the peaks of $A$ are the positions where the galaxy distribution resembles more the expected one 


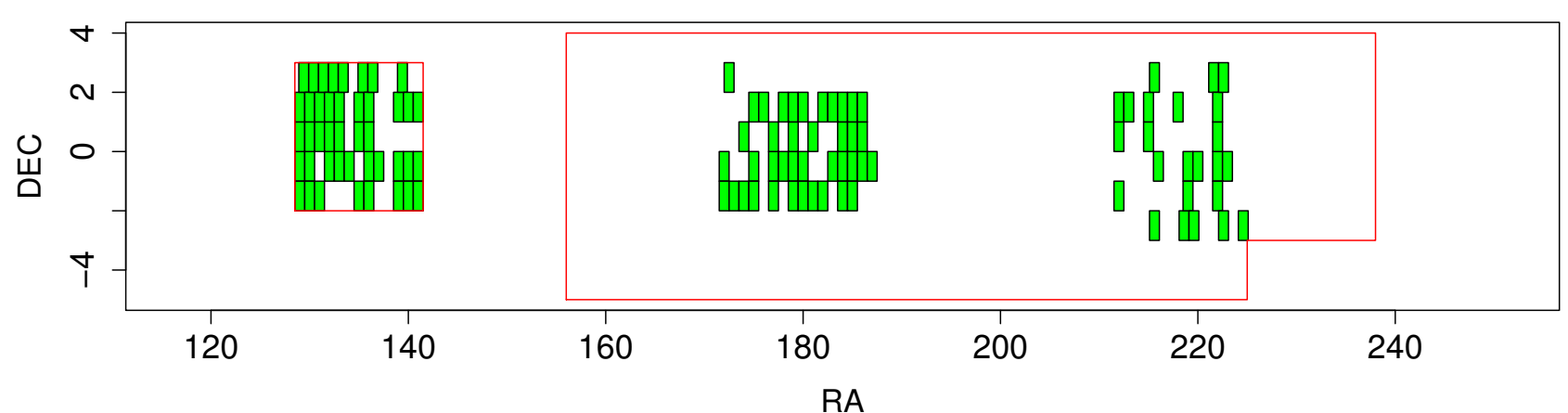

Fig. 1. Position on the sky of the KiDS-N ESO-DR2 tiles (in green). The red boxes show the total KiDS-N planned area.

for the cluster and is less likely to be due to random fluctuations of the background.

In this work, the model $M$ for the cluster is the expected galaxy distribution as a function of radius and magnitude in the $r$ band. This has been constructed from the average properties of clusters with mass $\sim 10^{14} M_{\odot}$ in the MaxBCG sample, which is derived from SDSS observations (Hansen et al. 2009; Sheldon et al. 2009; Hao et al. 2009). The background field distribution is conservatively estimated using the mean density of galaxies as a function of magnitude and redshift in the KiDS data.

For each tile, the amplitude $A$ is measured on a $3 \mathrm{D}$ grid which spans $\alpha, \delta$, and $z$ with a resolution of $\sim 250 \mathrm{kpc}$ spatially and 0.01 in redshift. The resolution in redshift is smaller than the typical uncertainty of the photometric redshifts, in order not to lose any information on the $z$ dimension present in the data. Then, the peaks of this map are detected and their signal-to-noise ratio $(\mathrm{S} / \mathrm{N})$ is calculated, dividing $\mathrm{A}$ by its uncertainty due to the fluctuations in the background and in the cluster galaxy population (see Eq. (9) in B11). In order to avoid multiple detections of the same halo, we build a cylindrical region around each significant peak, following the size-richness relation of Hansen et al. (2009). All the peaks at lower $\mathrm{S} / \mathrm{N}$ inside this region are considered "fragments" of the same halo and thus they do not enter the final catalog.

The redshift PDF enables us to weight each galaxy's contribution to the field and cluster components, and assign to each galaxy $i$ the probability $P_{i, j}$ to be a member of the cluster $j$ as

$P_{i, j}=\frac{A_{j} M\left(\boldsymbol{\theta}_{i}-\boldsymbol{\theta}_{j}, m_{i}\right) p_{i}\left(z_{j}\right)}{A_{j} M\left(\boldsymbol{\theta}_{i}-\boldsymbol{\theta}_{j}, m_{i}\right) p_{i}\left(z_{j}\right)+N\left(m_{i}, z_{j}\right)}$.

For each member galaxy, we can finally derive its best-fit template by running BPZ again, with the redshift fixed to the cluster redshift.

\section{Contamination and completeness tests}

\subsection{Contamination}

The optimal threshold for the $\mathrm{S} / \mathrm{N}$ should be chosen so that it maximizes the number of true detections (completeness) and minimizes the number of spurious detections (contamination). Spurious detections can originate either from galaxies randomly grouped together along the line of sight and mimicking a cluster due to the limited accuracy of photometric redshifts, or from real galaxy associations that are not actually genuine clusters (e.g., groups). In the following, we do not consider any detection associated with physical galaxy groups as spurious, even if its mass is significantly smaller than what is usually considered a cluster.
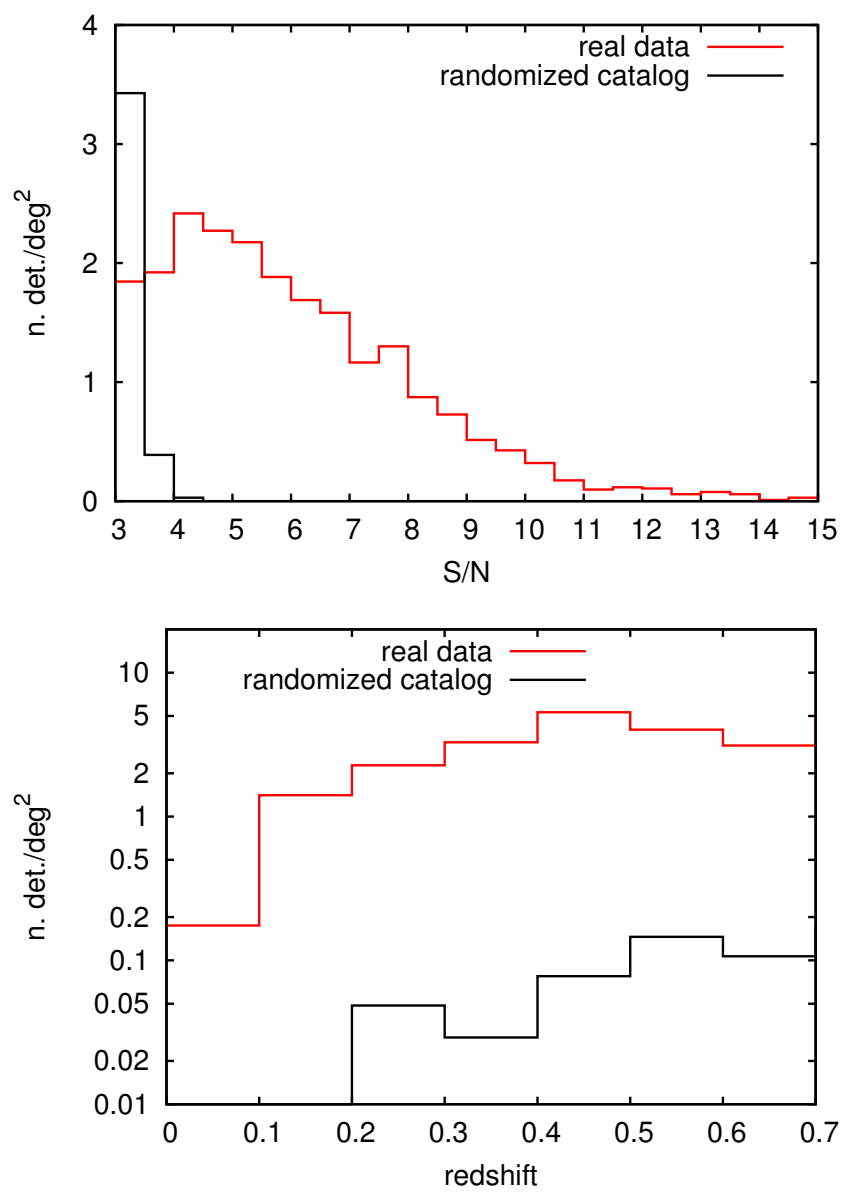

Fig. 2. Number of detections on real data and on randomized catalogs as a function of signal-to-noise ratio (top panel) and redshift (bottom panel). In the bottom panel, only detections with $S / N>3.5$ are shown, as this is the limit applied in the final analysis.

We aim at finding the optimal threshold that minimizes the detection of structures that are produced by random over-densities of objects along redshift and have no physical association.

To this end, we took each tile and randomized the positions of the galaxies in these regions. In this way, we obtained a catalog that retains all the observational properties of the original dataset (e.g., $p(z)$ distribution, mean density, and luminosity function), but where the structures have been erased. Thus, any possible detection in such a catalog is by definition a spurious detection. For each randomized catalog, we ran the cluster search as described in the previous section and analyzed the number of detections as a function of $\mathrm{S} / \mathrm{N}$. The results are shown in Fig. 2. From the top panel, one can see that in the randomized 
catalogs there is a relatively large number of detections produced by chance over-densities of objects, however few of them have $S / N>3.5$. In real data, there is a high probability that detections at low $\mathrm{S} / \mathrm{N}$ are considered as fragments of large structures, as described in Sect. 3; therefore, these detections are probably overestimated in the randomized catalogs compared to real data. Nevertheless, based on this test we selected $S / N=3.5$ as the optimal threshold, giving a contamination of $\sim 20 \%$; with a similar approach, a threshold $S / N=3$ was derived in B11. In the bottom panel of Fig. 2, we show the redshift distribution of these spurious detections after the cut in $\mathrm{S} / \mathrm{N}$ is applied. We did not consider here the contribution due to correlation from the large scale structure, which may increase the number of spurious detections.

\subsection{Completeness}

In principle, the completeness of the cluster-finding algorithm should be determined using mock galaxy clusters. By analysing the detection output, one can obtain an estimate of the completeness of the catalog as a function of redshift, thus providing the characterization of its selection function. However, as described in Ascaso et al. (2014), for example, the semi-analytic galaxy formation models currently available do not yet fully represent the photometric properties of galaxies in clusters. For these reasons, we adopted an alternative approach; we extracted clusters identified at high confidence from the survey itself, shifted them in redshift, and inserted them into the observed galaxy background population to study their detectability.

In detail, we identified two sets of candidate clusters at low redshift $\left(0.1<z_{\mathrm{c}}<0.3\right)$; a first sample of 19 objects at high $\mathrm{S} / \mathrm{N}$ $(S / N>6)$ that also match the redMaPPer catalog (see Sect. 6), and a second sample of 10 candidate clusters with low $\mathrm{S} / \mathrm{N}$ $(4.8<S / N<6)$. For each cluster, we created its "mock version" by considering its potential cluster members (galaxies with $P_{i, j}>0$, see the definition in Sect. 3) and applying a MonteCarlo method to define its actual members; for each ith potential member we independently extracted a random number $a_{i}$ between 0 and 1, and only included the galaxy in the mock cluster if $P_{i, j}>a_{i}$.

In order to model the star-formation history of each mock member, we considered their BPZ library classification, consisting of four CWW templates (Coleman et al. 1980) complemented by two starburst galaxy SEDs computed with GISSEL (Bruzual A. \& Charlot 1993). These six templates are well fitted by an exponentially declining star-formation history with an age of $13,10,5,5,10$, and $5 \mathrm{Gyr}$ and $\tau$ (the scale factor of the exponential) of $0.1,1.0,3.0,5.0,10$, and 10, respectively. Each mock galaxy was assumed to have an age and $\tau$ corresponding to its BPZ template at the redshift of the detection $z_{\mathrm{c}}$; this allowed us to de-evolve their SEDs using the Bruzual A. \& Charlot (1993) recipes, and determine their $r$-band magnitude at the redshift of our choice. We spanned the redshift range $0.2 \leq z \leq 0.75$ with 12 points $(\mathrm{d} z=0.05)$, thus creating a total of 348 mock clusters (228 for the high $\mathrm{S} / \mathrm{N}$ sample and 120 for the low one). It is important to stress that with our approach, the number of input galaxy members is constant with redshift, while the number of detected galaxy members varies according to the magnitude evolution.

Then, after choosing a random location in the tile as the mock cluster center, we removed the galaxies that fell below the magnitude limit of the tile, and placed the remaining members conserving the cluster geometry, that is, its physical size, and correcting the angular relative distances accordingly. Each cluster was also randomly rotated around its center. We choose the same tile of the original cluster detection to ensure that the galaxy selection function is the same. We avoid putting the cluster center too close to the map border $(10 \mathrm{arcmin})$ and to the original cluster center (12 arcmin).

As a last step, we need to assign a redshift probability distribution $p(z)$ to each galaxy. This property must be coherent to i) the "true" redshift $z_{\text {gal }}$ at which we are placing the galaxy; ii) its magnitude $r_{\text {gal }}$; iii) its BPZ template and iv) the properties of the $p(z)$ of similar galaxies in the KiDS data. For this purpose, we need a catalog of galaxies with known spectroscopic redshifts, covering the same redshift and magnitude range as KiDS images. To achieve this, we used data from the VST-SUDARE/VOICE survey (De Cicco et al. 2015) covering the COSMOS field. This survey is deeper than KiDS, therefore we selected a subset of images so that the final depths are the same as in KiDS. The images were processed in the AstroWISE system; ugri-band magnitudes, photometric redshifts and best-fit templates were obtained in the same way as for the KiDS data. Catalogs were then matched with the zCOSMOS-bright catalog ${ }^{2}$ DR3, providing spectroscopic redshifts for $\sim 20000$ galaxies $\left(i_{\mathrm{AB}}<22.5 \mathrm{mag}\right)$ in the COSMOS field. We thus obtained a catalog of $\sim 7000$ spectroscopic galaxies with redshift $0<z<1$.

For any given $z_{\text {gal }}$, we extracted a subsample of spectroscopic galaxies with the same BPZ template, and whose spectroscopic redshift $z_{\text {spec }}$ satisfied the relation $\left|z_{\text {spec }}-z_{\text {gal }}\right|<0.01$. When only a few spectroscopic galaxies satisfied this criterion, we increased the tolerance until we reached at least 20 of them: this happened only for some templates at high redshift, but we never had to increase the tolerance beyond 0.1. Once this subsample was defined, we randomly picked one galaxy with $\left|r-r_{\text {gal }}\right|<0.1$ and assigned its $p(z)$ to the mock galaxy. When no galaxy satisfied the latter criterion, we chose the one with the closest magnitude. This procedure ensures that we respect the items i)-iv) stated above.

Once this was done, we ran our algorithm again and checked if we detected the mock clusters and, in such a case, the $\mathrm{S} / \mathrm{N}$ of the detection. The results are shown in Fig. 3. Only a small number of simulated clusters (33 over 228) were missed by our detection algorithm for the high $\mathrm{S} / \mathrm{N}$ sample. This suggests a completeness of approximately $85 \%$ over the whole redshift range. When considering the lower $\mathrm{S} / \mathrm{N}$ sample, the completeness decreases to $70 \%$. We verified that these non-detections are due to the presence of higher $\mathrm{S} / \mathrm{N}$ detections nearby that masked them out (see the details in Sect. 3). On the other hand, when the detections are present, the high $\mathrm{S} / \mathrm{N}$ sample shows almost no evolution of the $\mathrm{S} / \mathrm{N}$ with redshift, with an increase from $z=0.2$ to $z=0.45$ by approximately 20 per cent and a decrease by the same amount down to $z=0.75$. This is the result of a combination of several factors, that include geometry and the difference between the redshift evolution of field and cluster galaxies. On the other hand, no significant trend with redshift is found for the low $\mathrm{S} / \mathrm{N}$ sample. For this sample, we can also observe that a small bias in the determination of the $\mathrm{S} / \mathrm{N}$ of our mock clusters is present that causes their average $\mathrm{S} / \mathrm{N}$ to be slightly higher than the original one at $z \sim 0.2$, obtained in real data. This is due to the combination of two effects. First, when computing the average, we consider only mock clusters that have actually been detected, thus with a higher chance of being at higher S/N. In addition, we verified that our subsample of galaxies with spectroscopic redshift have photometric redshift measurement slightly more

\footnotetext{
2 http://www . eso.org/sci/observing/phase3/data_ releases/zcosmos_dr3_b2.pdf
} 


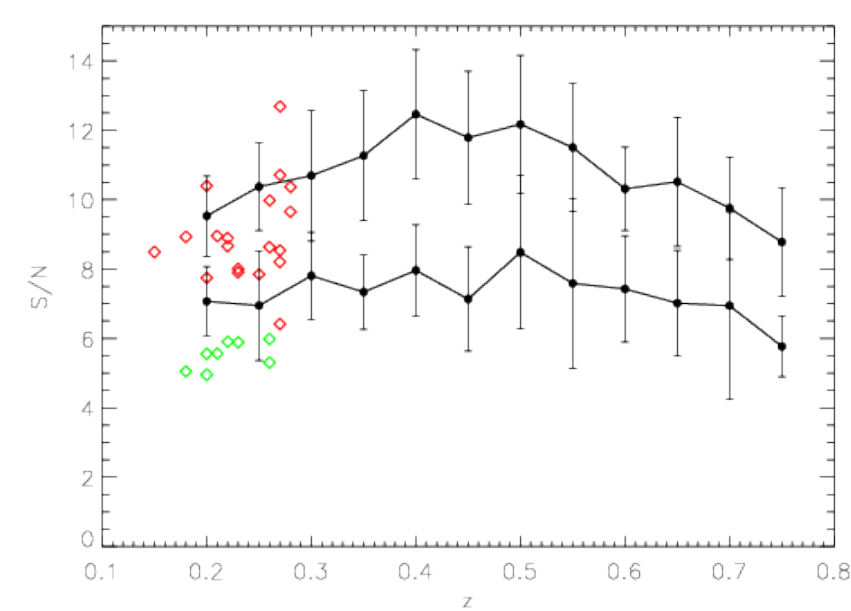

Fig. 3. Average signal-to-noise ratio for our mock clusters as a function of redshift, computed separately for the sample of halos originally detected in the real KiDS data with $S / N>6$ and $S / N<6$ (red and green points, respectively). For each sample, black lines show the average $S / N$ of the detected mock halos at a given redshift, while error bars indicate the rms.

precise than the average KiDS data at faint magnitudes: this likely induces a small increase of the $\mathrm{S} / \mathrm{N}$ of our mock haloes.

To summarize, our mock clusters do not change their $\mathrm{S} / \mathrm{N}$ significantly in the considered redshift interval and therefore we do not expect changes in the completeness up to $z \simeq 0.75$.

\section{Membership, richness, and mass}

Cluster members were selected as those galaxies with $P_{i, j}>0.2$ : this threshold excludes those galaxies that are too faint $(r>$ $24 \mathrm{mag})$ and/or too distant from the cluster center $(D>5 \mathrm{Mpc})$. The brightest galaxy (BCG) is defined as the one located within $0.5 \mathrm{Mpc}$ of the cluster center derived by the cluster finding procedure.

It is well known that the mass of clusters is well correlated with their total luminosity and richness (Koester et al. 2007; Andreon \& Hurn 2010). Andreon (2015) showed that the richness $(N)$ provides a good and reliable proxy to measure the mass $(M)$ :

$\log M_{\Delta}=\alpha+\beta\left(\log N_{\Delta}-2.0\right)+\gamma \frac{1+z}{1.15}$,

where $\Delta$ is the ratio between the cluster average mass density within a radius $R_{\Delta}, \rho_{\mathrm{c}}$ is the critical density of the Universe at that redshift (e.g., $\Delta=200$ or 500) and $N_{\Delta}$ is the richness derived as outlined below. The term describing the redshift dependence is small $(\gamma \sim-0.1)$. In their analysis, Andreon (2015) adopted the iterative approach proposed by Kravtsov et al. (2006). An initial value of $R_{\Delta}$ was chosen (e.g., $R_{\Delta}=1 h^{-1} \mathrm{Mpc}$ ), the richness $N_{\Delta} \equiv N\left(<R_{\Delta}\right)$ was computed and hence the mass from Eq. (5), assumed to be scatterless. Then, a new value of $R_{\Delta}$ was derived, according to:

$M_{\Delta}=\frac{4 \pi}{3} R_{\Delta}^{3} \times \Delta \times \rho_{\mathrm{c}}$.

The procedure was repeated until convergence. In Andreon (2015), the calibration of Eq. (5) was based on an X-ray selected sample of 39 clusters with masses derived by the caustics technique (Rines et al. 2013). An extended catalog of 275 clusters with richness-based masses was presented in Andreon (2016).
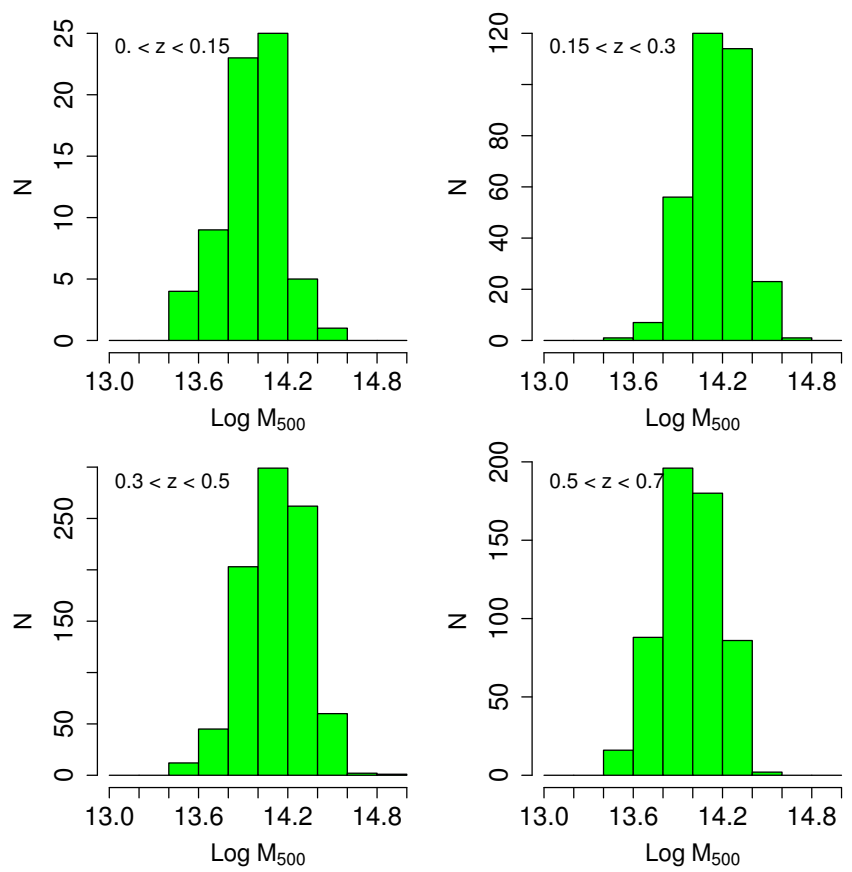

Fig. 4. Distribution of $M_{500}$ derived for the KiDS clusters in different redshift bins.

Since these clusters are not covered by the KDR2 tiles and no similar sample is yet available for the current KiDS area, we proceeded as follows. We started from the cluster catalog published by Wen \& Han (2015, WHL15 hereafter), that provides $R_{500}, R_{200}$ for 132684 clusters, using a richness proxy calibrated from a sample of 1191 clusters with masses estimated by X-ray or Sunyaev-Zeldovich measurements. By comparing the positions of these clusters with the final area available when KiDS will be completed, it turned out that 77 of these 1191 clusters will be detectable; presently however, only three of them fall into the KDR2 tiles, a number too low to produce a reliable fit. We therefore used the mass values derived for 230 clusters in the "full" WHL15 catalog for the calibration, for which $N_{500}>10$, $z_{\text {WHL15 }}-z_{\text {KiDS }}<0.05$. In this first step, $n_{\Delta}$ was defined as the number of KiDS cluster members located within a distance from the cluster center $R<R_{\Delta \text {,WHL15 }}$ and $r$-band absolute magnitudes $M_{\mathrm{abs}}+1.16 z<-20.5 \mathrm{mag}$, as in WHL15. $M_{200}$ and $M_{500}$ were derived from Eq. (6) with the values of $R_{200}$ and $R_{500}$ in the WHL15 catalog. The fit of Eq. (5) produced best-fit coefficients of: $\alpha=14.79 \pm 0.04, \beta=0.7 \pm 0.1(\Delta=500) ; \alpha=14.81 \pm 0.03$, $\beta=0.8 \pm 0.1(\Delta=200)$; we did not consider the small redshiftdependent term $(\gamma=0)$. Using these coefficients, we finally applied the iterative approach outlined before, and obtained $R_{\Delta}, N_{\Delta}$, and $M_{\Delta}$ for all KiDS clusters. We did not correct our mass estimate for selection effects such as the Eddington bias (see e.g., Mortonson et al. 2011; Sereno \& Ettori 2015): a more detailed discussion on the usage of our catalog for cosmological studies is deferred to the next paper, where more accurate mass estimates based on a larger area will be available.

\section{Results}

We applied the cluster search algorithm to the KDR2 tiles in the KiDS-N area. This gives an effective area of 114 sq. degrees, where we detected 1858 clusters with $0<z_{\mathrm{c}}<0.7, S / N>3.5$, and a mass $M_{500}$, derived as outlined in Sect. 5 , between $10^{13.5}$ and $\sim 7 \times 10^{14} M_{\odot}$ (Fig. 4). 
For each cluster we also computed the fraction $f$ of area lost due to masking or to the cluster proximity to the tile borders. The effective area $A_{\mathrm{c}}(R)$ within a given radius is thus: $A_{\mathrm{c}}(R)=f \pi R^{2}$. The richness values are corrected by this factor. We selected a subset of 1543 clusters with $f>0.9$ for which we provide a catalog with the following quantities (Table B.1): the cluster center given by the filter $\left(\boldsymbol{\theta}_{\mathrm{c}}\right)$; the redshift $\left(z_{\mathrm{c}}\right)$; the signal to noise ratio of the detection $(\mathrm{S} / \mathrm{N})$; the magnitude $\left(r_{\mathrm{BCG}}\right)$ and position $\left(\boldsymbol{\theta}_{\mathrm{BCG}}\right)$ of the brightest galaxy (the latter is hereafter assumed as the cluster center); the $R_{500}$ and $R_{200}$ radii, the $N_{500}$ and $N_{200}$ richness, and the $M_{500}$ and $M_{200}$ mass.

\section{Comparison with SDSS cluster catalogs}

In this section, we compare the KiDS clusters with those detected in the SDSS in the same area. We use three cluster catalogs derived using different cluster finder algorithms:

1. the redMaPPer catalog (Rykoff et al. 2014, RM hereafter), based on a red-sequence cluster finder;

2. the AMF catalog (Szabo et al. 2011), where clusters are identified by an adaptive matched filtering technique (Dong et al. 2008), similar to what was discussed in Sect. 3;

3. the WHL catalog first introduced in Wen et al. (2009) and updated in Wen et al. (2012), Wen \& Han (2015, WHL15), where clusters are selected using a friend-of-friends algorithm in the (RA, Dec, photo-z) space.

The number of clusters detected by each method depends strongly on the underlying assumptions, as well as on the adopted criteria (e.g., definition of membership, lower limit on richness, center definition). However, while a comparison does not enable us to assess the purity or completeness of a given algorithm, it is helpful to check the consistency of the physical parameters that are derived (redshift, mass, radius).

As a first step, we selected the clusters that fall within the KiDS tiles from the above SDSS-based catalogs. They were then matched to our catalog, pairing those clusters for which Szabo et al. (2011) centers are closer than $1 h^{-1} \mathrm{Mpc}$ and the difference in redshift is $\Delta z /(1+z) \leq 0.1$, considering that the KiDS photo- $z$ rms scatter is $\sim 0.05$ (see Sect. 2 ). The match was done in such a way that each cluster from one catalog was matched to only one cluster from the other catalog; in case of multiple matches, the nearest neighbour in both redshift and position is chosen. Table 1 and Fig.5 summarize the number of clusters found in the RM, AMF, and WHL15 catalogs ( $\left.N_{\text {SDSS }}\right)$, and of those matched by KiDS clusters $\left(N_{\mathrm{m}}\right)$; the last column gives the fraction of matched clusters with separation below $10^{\prime \prime}$. The redshift distribution of KiDS and SDSS clusters is displayed in Fig. 6, which also shows the fraction of matched clusters in different redshift bins.

The unmatched clusters can be either real clusters not recovered by our algorithm or spurious detections in the SDSSbased catalogs, or due to an incorrect redshift estimate. An additional issue arises due to the way in which each algorithm handles nearby clusters, merging them in one cluster, or keeping separate substructures. For instance, Szabo et al. (2011) conclude that, compared to their AMF catalog, the WHL algorithm presented in Wen et al. (2009) produces a fragmentation of the largest clusters into several small clusters. Figure 7 compares the number of matched and unmatched clusters vs. the richness defined in the RM, AMF, and WHL15 catalogs, and the fraction of matched clusters as a function of the KiDS richness $\left(N_{500}\right)$. In general, the matching fraction decreases with increasing redshift $\left(z>0.3\right.$, Fig. 6) and decreasing richness $\left(>70 \%\right.$ for $N_{500}>40$,
Table 1. Comparison of candidate clusters from SDSS-based catalogs in KiDS tiles and KDR2 candidate clusters.

\begin{tabular}{cccccc}
\hline \hline Source & $N_{\text {SDSS }}$ & $N_{\mathrm{m}}$ & $N_{\mathrm{m}} / N_{\text {SDSS }}$ & $N_{\mathrm{m}} / N_{\text {KiDS }}$ & $\underset{\prime \prime}{\text { Sep }}$ \\
\hline RM & 293 & 226 & $77 \%$ & $12 \%$ & 27 \\
AMF & 1029 & 593 & $58 \%$ & $32 \%$ & 87 \\
WHL15 & 1241 & 639 & $51 \%$ & $34 \%$ & 29 \\
\hline
\end{tabular}

Notes. $N_{\text {SDSS }}$ is the number of SDSS candidates, $N_{\text {KiDS }}(1858)$ is the number of KiDS candidates found with our method and $N_{\mathrm{m}}$ are those matched, by position and redshift, among our and SDSS based catalogs. The last column shows the median separation in the centers.

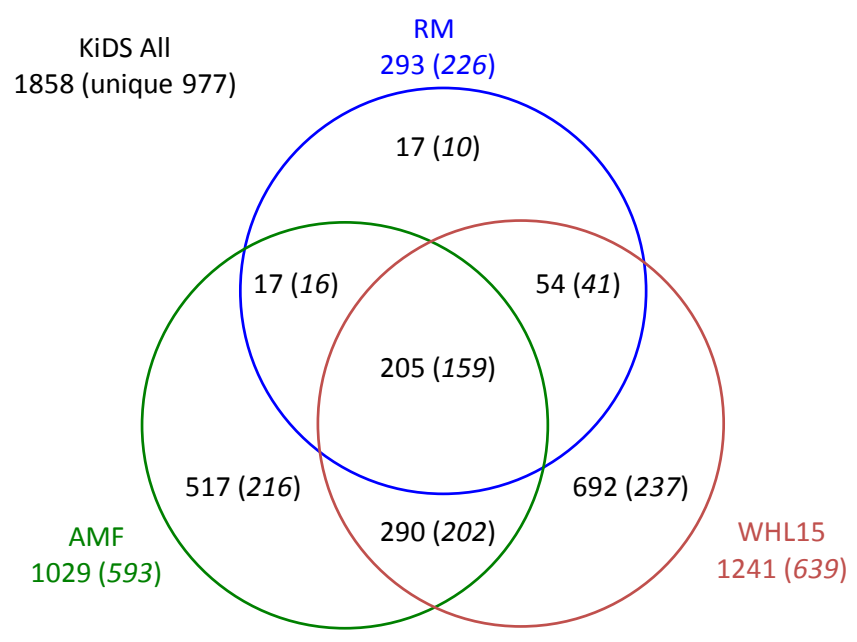

Fig. 5. Venn diagram showing the number of clusters matched among the SDSS-based catalogs in the KDR2 footprint; the numbers of clusters also matched in KiDS are displayed within brackets.

$<40 \%$ for $N_{500}<30$, Fig. 7), where defining a cluster is more difficult. A similar result is found by Szabo et al. (2011), when comparing the AMF and WHL (Wen et al. 2009) catalogs.

\section{Comparison with RM}

The RM catalog (Rykoff et al. 2014) was derived by applying redMaPPer, a red-sequence cluster finder algorithm, to $\sim 10000 \mathrm{deg}^{2}$ in the SDSS DR8: it consists of approximately 25000 clusters with masses $>10^{14} M_{\odot}$ in a redshift range $0.08 \leq$ $z \leq 0.55$. The cluster catalog contains the cluster sky position, redshift, and the richness estimate $(\Lambda)$ and includes only clusters with redshift $z>0.1$ and a richness $\Lambda>20$, below which the cluster completeness is shown to be lower than 50\%. A separate catalog provides the galaxies identified as members of each cluster (coordinates, membership probability and de-reddened magnitudes).

A catalog of galaxies that are likely cluster red members is also available; this enabled us to select the RM clusters for which at least $80 \%$ of the galaxy members are also detected in $\mathrm{KiDS}$, and reject RM clusters located close to the borders of KiDS tiles, or on masked regions. After this selection, we obtain 293 RM clusters in our area. We find that 77\% (226/293) of RM clusters are also found in KiDS. Of the 226 matched clusters, the separation is $<5$ arcmin for $\sim 99 \%$ of them, with a median separation of $\sim 27$ arcsec.

Figure 8 compares KiDS with RM cluster redshifts; selecting those clusters for which the redshift is $z<0.5$, we obtain 

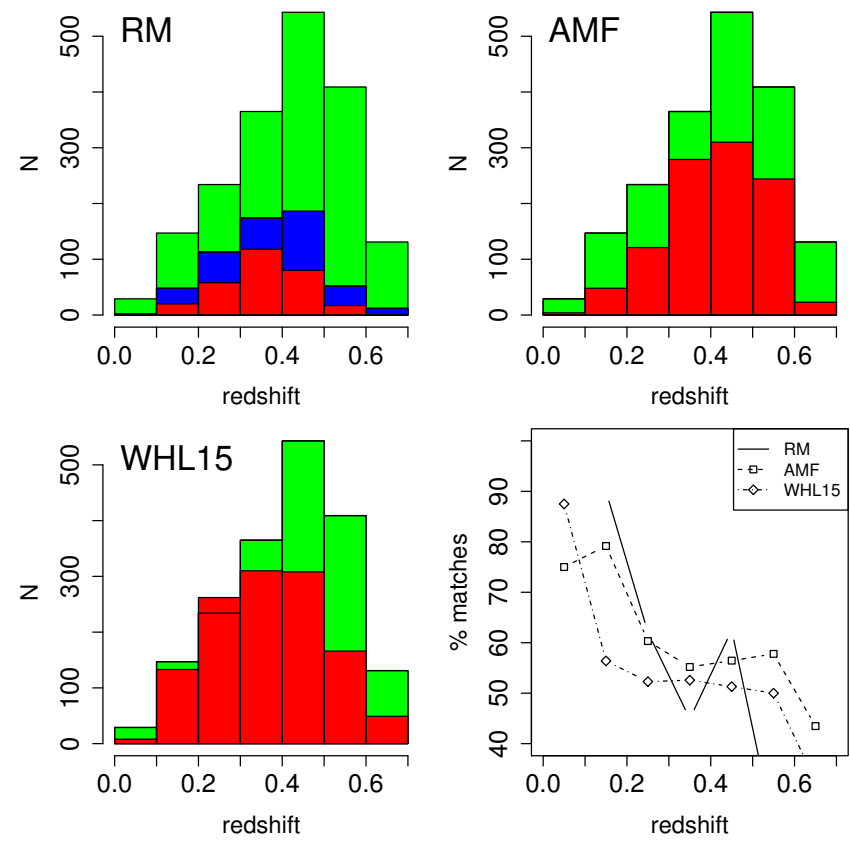

Fig. 6. First three panels: redshift distribution of all cluster candidates in the RM, WHL15 and AMF cluster catalogs within the KiDS tiles (red), compared with the KiDS clusters (green). In the first panel (RM), also displayed are the KiDS clusters with a number of early-type galaxies $n_{\mathrm{et}}>20$ (blue). Last panel: fraction of matches only as a function of redshift.
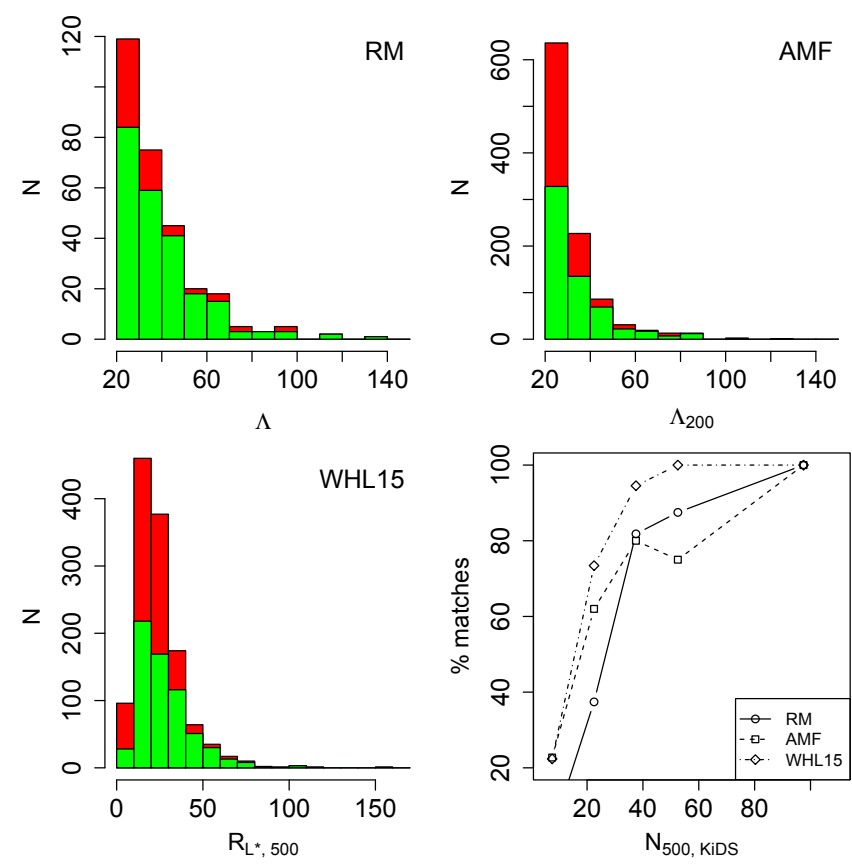

Fig. 7. Distribution of richness estimates for cluster candidates in the $\operatorname{RM}(\Lambda), \operatorname{AMF}\left(\Lambda_{200}\right)$ and WHL15 $\left(R_{L *, 500}\right)$ catalogs (in red), with overlaid those matched by KiDS clusters (in green). Last panel: fraction of matches as a function of the KiDS $N_{500}$ richness.

$\sigma(\Delta z /(1+z))=0.02$ for both RM photometric and spectroscopic redshifts.

The redshift distributions of the RM (in red) and KiDS clusters (green: all KiDS clusters) are compared in Fig. 6: we detect a significantly higher number of clusters in KiDS than in RM at all redshifts. In order to understand the reason for this difference, we extracted a subsample of KiDS cluster member galaxies for which the best-fit template corresponds to CWW early-type
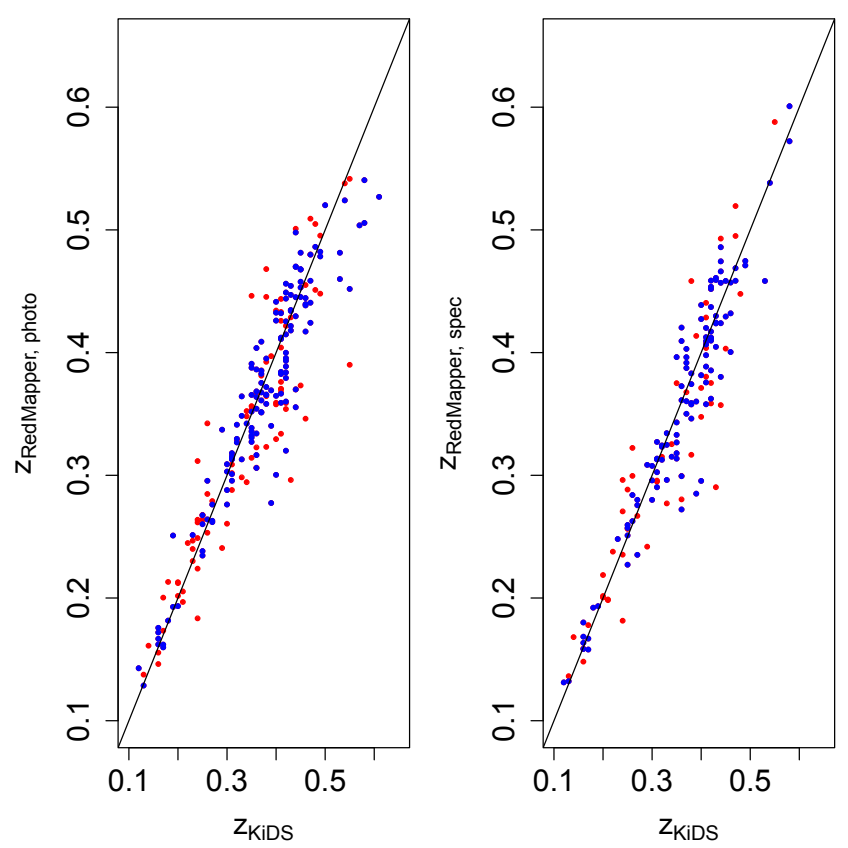

Fig. 8. Comparison of KiDS cluster redshifts with RM photometric (left) and spectroscopic (right) redshifts. Red dots are clusters matched within 3 arcmin and clusters matched within 1 arcmin are displayed in blue.

galaxies, galaxies that are located within $1 \mathrm{~h}^{-1} \mathrm{Mpc}$ from the cluster center, and galaxies that have an $i$-band magnitude brighter than $m_{*}+1.75$ mag (Rykoff et al. 2014). In this way, we define a richness $n_{\text {et }}$ that can be used for comparison with the richness derived in redMaPPer. We then selected those clusters with $n_{e t}>20$; the median $\mathrm{S} / \mathrm{N}$ for the clusters below this limit is approximately 6 . The distribution obtained after this cut is displayed in blue in Fig. 6, showing that the RM and KiDS distributions are now much closer, up to $z \sim 0.3$. The number of KiDS vs. RM clusters increases at higher redshifts, as expected due to the different depths of the parent datasets.

\section{Comparison with AMF}

The AMF catalog consists of 69173 clusters in the redshift range $0.045 \leq z<0.78$ covering an area $\sim 8420 \mathrm{deg}^{2}$ from SDSS DR6: for each cluster, it provides the position of the cluster center and its redshift, the richness estimate $\left(\Lambda_{200}\right)$, defined as the total luminosity in units of $L^{*}$ within the radius $R_{200}$. Cluster centers are defined as the position that maximizes the cluster detection probability along a grid of resolution $1 h^{-1} \mathrm{kpc}$ around the initial position. Clusters are only included in the catalog if their richness is $\Lambda_{200}>20$, which should produce a completeness $\sim 85 \%$ for clusters with $M_{200}>10^{14} h^{-1} M_{\odot}$, based on simulations (Dong et al. 2008). A catalog of the three brightest galaxies in the $r$ band in each cluster ( 205000 galaxies) is also available.

1029 AMF clusters are included in the KDR2 area. Of these, $593(58 \%)$ are matched by KiDS clusters. The AMF radius $\left(R_{200}\right)$ and richness $\left(\Lambda_{200}\right)$ show a good correlation with those derived for the KiDS clusters $N_{200}$ (Pearson correlations: $r=0.6$ and $r=0.7$ respectively). The distance between the centers of the AMF clusters and the KiDS clusters (Table 1) is larger than that found for the RM. This is due to the cluster center definition adopted in AMF, which is not related to a BCG galaxy, as in RM, WHL15, and in our case. 


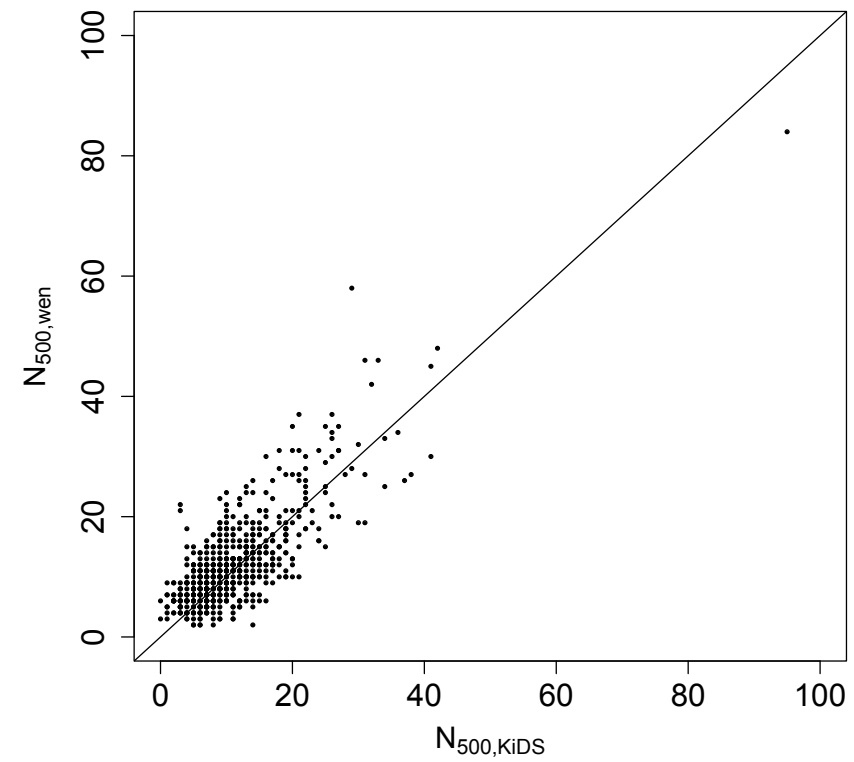

Fig. 9. Comparison between the number of member galaxies found within $R=R_{500, \mathrm{WHL} 15}$, in KiDS vs. WHL15. The line displays the bisector as reference.

\section{Comparison with WHL15}

The WHL15 catalog consists of 132684 clusters in the redshift range $0.05 \leq z \leq 0.8$ from SDSS DR12, providing the sky position of the BCG, which defines the cluster center, and its $r$-band magnitude, the cluster redshift, the radius $R_{500}$ and the number of cluster members within this radius $\left(N_{500}\right)$, and the richness estimate $\left(R_{L *, 500}\right)$. The scaling relations between the total $r$-band luminosity of member galaxies within $1 \mathrm{Mpc}$ and the cluster radius were established using a sub-sample of 1191 clusters with mass derived by X-ray or Sunyaev-Zeldovich measurements. No richness cut is applied to the cluster catalog.

There are 1241 WHL15 clusters in our KiDS tiles, 639 (51\%) of which are matched in KiDS; for comparison, 226 (77\%) are matched by RM clusters. Of the 602 WHL15 clusters not found in KiDS, 59 are matched by RM clusters.

The comparison of $R_{500}, N_{500}$ for the clusters matched in WHL15 and KiDS gives a good correlation (Pearson correlations: $r=0.5$ and $r=0.6$ respectively). In order to compare the selection of member galaxies, we assumed the $r_{500}$ value from the WHL15 catalog for each cluster, and counted the KiDS member galaxies $r \leq r_{500}$ from the BCG. As in WHL15, we only further considered those galaxies with an absolute magnitude $M_{\text {abs }}(r)+1.16 z<-20.5$. The result is displayed in Fig. 9 .

Figure 10 compares the KiDS and WHL15 values of $M_{500}$ for the matched clusters; the red dots display $M_{500}$ for the three clusters from the WHL15 catalog of 1191 clusters with known masses. The Pearson correlation is $r=0.6$.

\section{Comparison with Abell, XMM, and Planck catalogs}

One way of making a crucial assessment of the reliability of our detection algorithm and of the cluster candidate catalog is to recover confirmed clusters included in the same area. To this end, cluster identifications were matched with the cluster catalogs of Abell (ACO, Abell et al. 1989), XMM Cluster Survey (XCS, Mehrtens et al. 2012), and Planck-SZ (PSZ1, Planck Collaboration XXIX 2014; Planck Collaboration XXXII 2015); a matching radius of $1.5 h^{-1} \mathrm{Mpc}$ was used here, to take

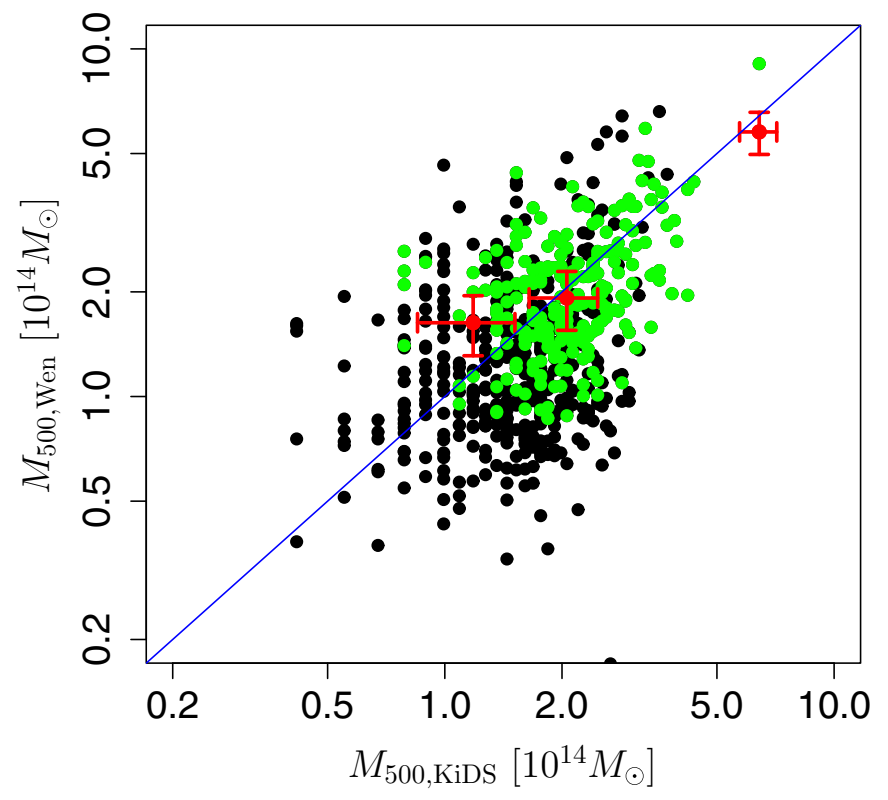

Fig. 10. Comparison of KiDS and WHL15 $M_{500}$ (in units of $10^{14} M_{\odot}$ ); green dots are the clusters used for the mass calibration. Red dots with error bars are the clusters found in the current KiDS area from the cata$\log$ of 1191 clusters in WHL15. The line is the bisector.

into account the larger uncertainties in the centers of the X-ray and PSZ1 clusters. Given the limited size of the KiDS area analyzed here, only a few of these clusters are found.

\section{Abell clusters}

In the KDR2 area, there are 11 Abell clusters; 9 of them are unambiguously matched by KiDS clusters, the separation between their centers being $<2^{\prime}$. Table A. 1 reports their properties (Abell et al. 1989; Abell 1958) and those derived here for the KiDS matched clusters. For the matching, we used the cluster centers listed in De Propris et al. (2002), when available, since they are more accurate than those given in Abell et al. (1989). For two clusters (Abell 1389 and Abell 1419), the matching is more uncertain, as the separation is large $\left(\sim 7^{\prime}\right)$. Abell $1389(z=$ 0.08) belongs to the Leo A supercluster (Einasto et al. 1997), that also includes Abell 1386, which may explain the uncertainty on the cluster center. In the case of Abell 1419, the redshift is significantly different $\left(z_{\mathrm{ACO}}=0.12, z_{\mathrm{KiDS}}=0.27\right)$. However, a cluster at the same position and redshift as the KiDS candidate is also found in WHL15 (WHL J115610.8-002101): this would imply that the detection of Abell 1419 is masked by a cluster at higher redshift.

The field around four Abell clusters is displayed in Fig. A.1.

\section{XMM clusters}

The XCS-DR1 (Mehrtens et al. 2012) consists of 503 optically confirmed clusters and includes estimates of $R_{200}$ and $R_{500}$ based on X-ray data (Lloyd-Davies et al. 2011), and photometric or spectroscopic redshifts from optical identifications. Four XCS clusters fall in the KDR2 area (Table A.2): all of them are matched within the criteria defined above. For at least three of them, their redshifts (based on the SDSS: two photometric, one spectroscopic) agree well with the values found in KiDS. No information on the accuracy of the redshift $(z=0.15)$ of 
XCS J1448.1-0025 (from Goto et al. 2002) is available, preventing a meaningful comparison with the KiDS redshift $(z=0.23)$.

\section{Planck-SZ clusters}

There are three Planck clusters (Planck Collaboration XXIX 2014; Planck Collaboration XXXII 2015) in the KDR2 area (Table A.3); taking into account the uncertainties on the cluster center given in the Planck catalog, the matching radius is more relaxed and therefore all Planck clusters present in our area can be considered to have a KiDS counterpart inside errors. The validation status parameter (Col. 4) provides a class of reliability for the new Planck detections. Two of our three Planck counterparts were also detected by Zwicky et al. (1968), while the third is a Planck candidate of intermediate level of reliability. Also displayed in Table A.3 are $M_{500}^{Y_{Z}}$, the mass derived from the SZ mass proxy, and $M_{500}$, the mass derived in Sect. 6, however, with only two clusters, no meaningful comparison can be made yet.

\section{Conclusions}

In this paper, we present the methods adopted for the detection of clusters in the KiDS survey, based on an optimal filtering technique, and the initial results from KDR2, giving a catalog of 1858 clusters with redshift $0<z<0.7$. The signal to noise threshold was selected by randomizing the position of galaxies and deriving the number of spurious detections as a function of $\mathrm{S} / \mathrm{N}$; we chose a threshold $S / N=3.5$ to minimize the number of incorrect identifications. The completeness $(\sim 85 \%)$ of the cata$\log$ is derived selecting clusters at low redshift from the data and simulating the effect of moving them to higher redshifts. An estimate of the mass at $R_{500}$ and $R_{200}$ is done using the richness as a proxy; this requires calibration of Eq. (5) for our data. The accuracy of this calibration is still limited; we expect to improve it when more clusters with accurate masses are observed within the KiDS area. We present the results of the comparison between our catalog and those derived on the SDSS in the same area, showing an agreement for $>50 \%$ of the clusters. Most of the candidate clusters present in the SDSS catalogs, but not found by us, are those with a low richness. A list of the clusters from the Abell, $\mathrm{XMM}$, and Planck-SZ surveys included inside the KDR2 tiles is presented in Sect. 7.

The results outlined in this paper are preparatory to building a statistically significant sample of clusters with richness and mass measurements. An area of 450 sq. degrees is now publicly available (KiDS ESO-DR3); the analysis of these new data for the cluster search is in progress, aiming to derive, for example, the mass function at different redshift bins and then to extract constraints on the main cosmological parameters.

Acknowledgements. M.Ra., F.B., L.M. and M.Ro. acknowledge the grants ASI No. I/023/12/0 "Attività relative alla fase B2/C per la missione Euclid" and MIUR PRIN 2010-2011 "The dark Universe and the cosmic evolution of baryons: from current surveys to Euclid". F.B., L.M. and M.Ro. acknowledge the grants PRIN INAF 2012 "The Universe in the box: multiscale simulations of cosmic structure". M.M. has been supported in part by the TransregioSonderforschungsbereich TR 33 of the Deutsche Forschungsgemeinschaft. M.B. is supported by the Netherlands Organization for Scientific Research, NWO, through grant number 614.001.451, and through FP7 grant number 279396 from the European Research Council. We thank the referee for his/her comments, which improved the paper.

\section{References}

Abell, G. O. 1958, ApJS, 3, 211

Abell, G. O., Corwin, Jr., H. G., \& Olowin, R. P. 1989, ApJS, 70, 1
Allen, S. W., Evrard, A. E., \& Mantz, A. B. 2011, ARA\&A, 49, 409

Andreon, S. 2015, A\&A, 582, A100

Andreon, S. 2016, A\&A, 587, A158

Andreon, S., \& Hurn, M. A. 2010, MNRAS, 404, 1922

Ascaso, B., Mei, S., \& Benitez, N. 2014, in SF2A-2014: Proc. of the Annual Meeting of the French Society of Astronomy and Astrophysics, eds. J. Ballet, F. Martins, F. Bournaud, R. Monier, \& C. Reylé, 299

Bellagamba, F., Maturi, M., Hamana, T., et al. 2011, MNRAS, 413, 1145 Benítez, N. 2000, ApJ, 536, 571

Bruzual A., G., \& Charlot, S. 1993, ApJ, 405, 538

Cavuoti, S., Brescia, M., Tortora, C., et al. 2015, MNRAS, 452, 3100 Cavuoti, S., Amaro, V., Brescia, M., et al. 2016, MNRAS, submitted Choi, A., Heymans, C., Blake, C., et al. 2016, MNRAS, 463, 3737 Coleman, G. D., Wu, C.-C., \& Weedman, D. W. 1980, ApJS, 43, 393 De Cicco, D., Paolillo, M., Covone, G., et al. 2015, A\&A, 574, A112 de Jong, J. T. A., Kuijken, K., Applegate, D., et al. 2013, The Messenger, 154, 44

de Jong, J. T. A., Verdoes Kleijn, G. A., Boxhoorn, D. R., et al. 2015, A\&A, 582, A62 (KDR2)

De Propris, R., Couch, W. J., Colless, M., et al. 2002, MNRAS, 329, 87

Dong, F., Pierpaoli, E., Gunn, J. E., \& Wechsler, R. H. 2008, ApJ, 676, 868

Driver, S. P., Hill, D. T., Kelvin, L. S., et al. 2011, MNRAS, 413, 971

Einasto, M., Tago, E., Jaaniste, J., Einasto, J., \& Andernach, H. 1997, A\&AS, 123,119

Eke, V. R., Cole, S., Frenk, C. S., \& Patrick Henry, J. 1998, MNRAS, 298, 1145 Estrada, J., Annis, J., Diehl, H. T., et al. 2007, ApJ, 660, 1176

Goto, T., Sekiguchi, M., Nichol, R. C., et al. 2002, AJ, 123, 1807

Hansen, S. M., Sheldon, E. S., Wechsler, R. H., \& Koester, B. P. 2009, ApJ, 699, 1333

Hao, J., Koester, B. P., Mckay, T. A., et al. 2009, ApJ, 702, 745

Henriques, B. M. B., White, S. D. M., Lemson, G., et al. 2012, MNRAS, 421, 2904

Hildebrandt, H., Viola, M., Heymans, C., et al. 2017, MNRAS, 465, 1454

Koester, B. P., McKay, T. A., Annis, J., et al. 2007, ApJ, 660, 239

Kravtsov, A. V., Vikhlinin, A., \& Nagai, D. 2006, ApJ, 650, 128

Kuijken, K., Heymans, C., Hildebrandt, H., et al. 2015, MNRAS, 454, 3500

Laureijs, R., Amiaux, J., Arduini, S., et al. 2011, ArXiv e-prints

[arXiv: 1110.3193$]$

Lloyd-Davies, E. J., Romer, A. K., Mehrtens, N., et al. 2011, MNRAS, 418, 14 Maturi, M., Meneghetti, M., Bartelmann, M., Dolag, K., \& Moscardini, L. 2005, A\&A, 442, 851

Mehrtens, N., Romer, A. K., Hilton, M., et al. 2012, MNRAS, 423, 1024

Melchior, P., Suchyta, E., Huff, E., et al. 2015, MNRAS, 449, 2219

Merchán, M., \& Zandivarez, A. 2002, MNRAS, 335, 216

Mortonson, M. J., Hu, W., \& Huterer, D. 2011, Phys. Rev. D, 83, 023015

Pace, F., Maturi, M., Bartelmann, M., et al. 2008, A\&A, 483, 389

Planck Collaboration XXIX. 2014, A\&A, 571, A29

Planck Collaboration XXXII. 2015, A\&A, 581, A14

Popesso, P., Biviano, A., Böhringer, H., \& Romaniello, M. 2007, A\&A, 461, 397

Quintana, H., \& Ramirez, A. 1995, ApJS, 96, 343

Rines, K., Geller, M. J., Diaferio, A., \& Kurtz, M. J. 2013, ApJ, 767, 15

Rykoff, E. S., Rozo, E., Busha, M. T., et al. 2014, ApJ, 785, 104 (RM)

Rykoff, E. S., Rozo, E., Hollowood, D., et al. 2016, ApJS, 224, 1

Sartoris, B., Biviano, A., Fedeli, C., et al. 2016, MNRAS, 459, 1764

Sereno, M., \& Ettori, S. 2015, MNRAS, 450, 3633

Sheldon, E. S., Johnston, D. E., Masjedi, M., et al. 2009, ApJ, 703, 2232

Springel, V., White, S. D. M., Jenkins, A., et al. 2005, Nature, 435, 629

Struble, M. F., \& Rood, H. J. 1999, ApJS, 125, 35

Szabo, T., Pierpaoli, E., Dong, F., Pipino, A., \& Gunn, J. 2011, ApJ, 736, 21 (AMF)

Tarrío, P., Melin, J.-B., Arnaud, M., \& Pratt, G. W. 2016, A\&A, 591, A39

The Dark Energy Survey Collaboration 2005, ArXiv e-prints [arXiv:astro-ph/0510346]

Verdoes Kleijn, G., de Jong, J. T. A., Valentijn, E. A., et al. 2011, ArXiv e-prints [arXiv: 1112.0886]

Wen, Z. L., \& Han, J. L. 2015, ApJ, 807, 178 (WHL15)

Wen, Z. L., Han, J. L., \& Liu, F. S. 2009, ApJS, 183, 197

Wen, Z. L., Han, J. L., \& Liu, F. S. 2010, ApJS, 187, 272

Wen, Z. L., Han, J. L., \& Liu, F. S. 2012, ApJS, 199, 34

White, S. D. M., Navarro, J. F., Evrard, A. E., \& Frenk, C. S. 1993, Nature, 366, 429

Yoon, J. H., Schawinski, K., Sheen, Y.-K., Ree, C. H., \& Yi, S. K. 2008, ApJS, 176,414

York, D. G., Adelman, J., Anderson, Jr., J. E., et al. 2000, AJ, 120, 1579

Zwicky, F., Herzog, E., \& Wild, P. 1968, Catalogue of galaxies and of clusters of galaxies (Pasadena: California Institute of Technology) 
Appendix A: Properties of Abell, XMM, and Planck clusters
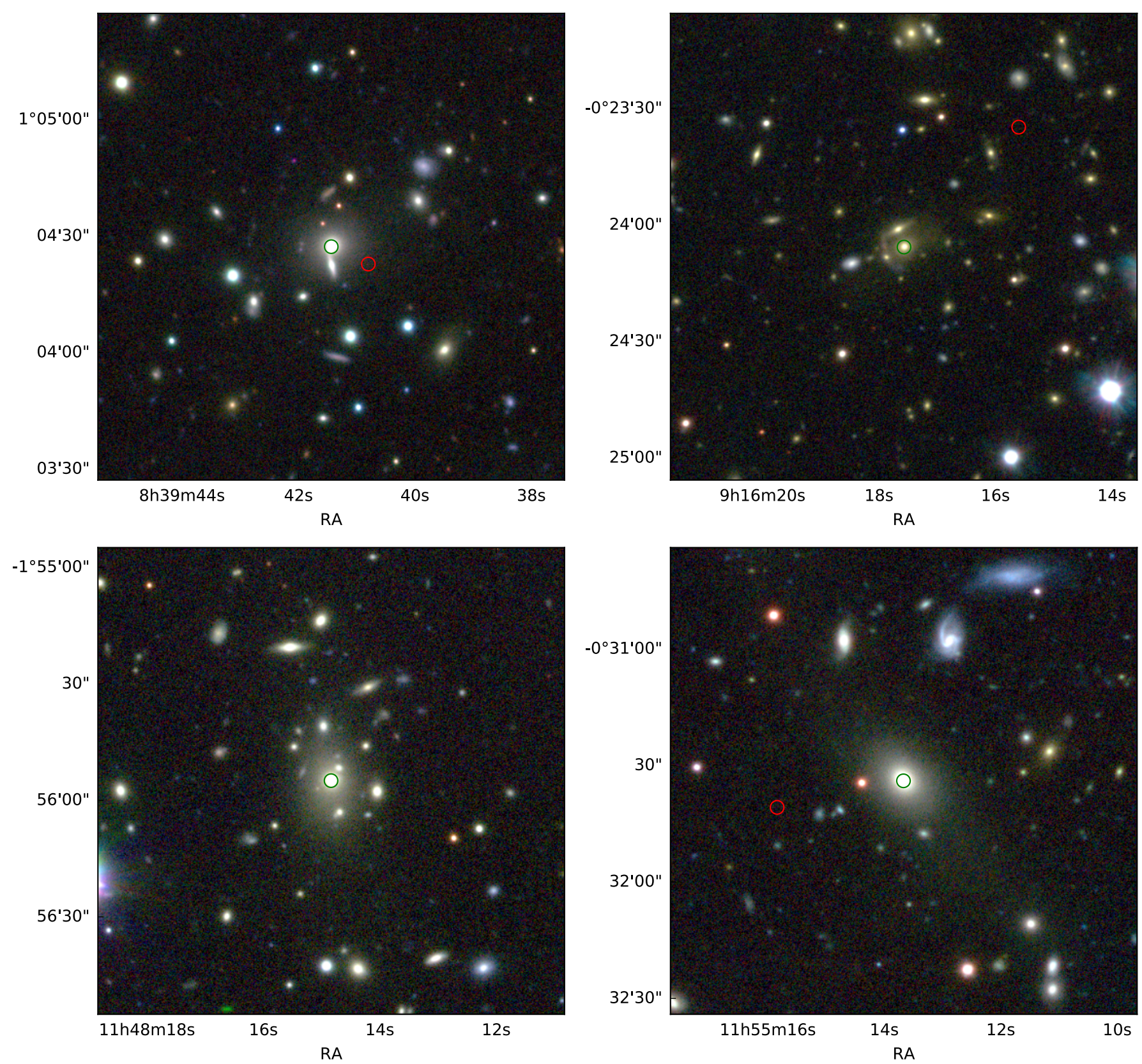

Fig. A.1. KiDS ugr images of the inner 2' regions for the Abell clusters A693, A776, A1386 and A1411 (from upper-left clockwise). Green circles show the position of the BCG; the cluster centers in the Abell catalog are displayed by the red circles, if within the region. 
M. Radovich et al.: Searching for galaxy clusters in the Kilo-Degree Survey

Table A.1. Matching between Abell (Abell et al. 1989) and KiDS clusters.

\begin{tabular}{|c|c|c|c|c|c|c|c|c|c|}
\hline \multirow[b]{2}{*}{ ID } & \multicolumn{4}{|c|}{ Abell } & \multicolumn{4}{|c|}{ KiDS } & \multirow[b]{2}{*}{ Sep. $\left({ }^{\prime \prime}\right)$} \\
\hline & $\mathrm{RA}^{*}$ & Dec $^{*}$ & Richness** & $z^{* * *}$ & RA & Dec & $z$ & $S / N$ & \\
\hline A693 & 129.920 & 1.073 & $46[0]$ & $0.16(5)$ & 129.923 & 1.074 & 0.17 & 7.9 & 11 \\
\hline A711 & 132.592 & 0.313 & $57[1]$ & 0.19 (6) & 132.600 & 0.260 & 0.19 & 10.6 & 192 \\
\hline A776 & 139.065 & -0.393 & 51 & $0.33(7)$ & 139.073 & -0.402 & 0.37 & 16.4 & 43 \\
\hline A1376 & 176.529 & -1.094 & $50[1]$ & 0.12 (1) & 176.564 & -1.096 & 0.12 & 8.9 & 126 \\
\hline A1386 & 177.090 & -1.945 & $66[1]$ & $0.10(2)$ & 177.062 & -1.932 & 0.18 & 9.4 & 112 \\
\hline A1411 & 178.816 & -0.528 & 69 & $0.13(3)$ & 178.807 & -0.526 & 0.15 & 8.5 & 33 \\
\hline A1445 & 180.431 & -0.184 & $81[2]$ & 0.17 (1) & 180.432 & -0.184 & 0.19 & 13.2 & 4 \\
\hline A1533 & 186.140 & 0.906 & 119 [2] & $0.23(1)$ & 186.122 & 0.898 & 0.25 & 7.2 & 73 \\
\hline A1938 & 219.432 & -0.316 & 53 & $0.14(4)$ & 219.433 & -0.316 & 0.14 & 10.1 & 2 \\
\hline A1389 & 177.281 & -1.367 & $40[0]$ & $0.08(8)$ & 177.187 & -1.284 & 0.12 & 6.6 & 452 \\
\hline A1419 & 179.083 & -0.206 & $73[1]$ & $0.12(3)$ & 179.041 & -0.326 & 0.27 & 12.7 & 457 \\
\hline
\end{tabular}

Notes. The last column (Sep.) gives the distance in arcsec between the centers of the Abell cluster and the KiDS cluster. ${ }^{(*)}$ RA and Dec for the clusters A1376, A1389, A1419, A1445 and A1938 are from De Propris et al. (2002). The others are from Abell et al. (1989). ${ }^{(* *)}$ Richness as defined in Abell et al. (1989) (number of cluster members between $m_{3}$ and $m_{3}+2$, corrected for background) and, in square brackets, richness class from Abell (1958). ${ }^{(* *)}$ Redshift from different references, indicated in brackets; (5), (6) and (7) are photometric redshifts.

References. (1) Struble \& Rood (1999); (2) Quintana \& Ramirez (1995); (3) Popesso et al. (2007); (4) Priv. comm., M. Merchan, and A. Zandivarez, unpublished Galaxy Groups catalog as described in Merchán \& Zandivarez (2002) and sent to Dr. John Mulchaey in 2003. Received by NED in 2007; (5) Koester et al. (2007); (6) Wen et al. (2010); (7) Estrada et al. (2007); (8) Yoon et al. (2008).

Table A.2. Matching between XCS (Mehrtens et al. 2012) and KiDS clusters.

\begin{tabular}{|c|c|c|c|c|c|c|c|c|c|c|c|c|}
\hline \multirow[b]{2}{*}{ ID } & \multicolumn{5}{|c|}{ XCS } & \multicolumn{6}{|c|}{ KiDS } & \multirow[b]{2}{*}{$\begin{array}{r}\text { Sep } \\
\left({ }^{\prime \prime}\right)\end{array}$} \\
\hline & RA & Dec & $z^{* *}$ & $\begin{array}{c}R_{500}{ }^{* *} \\
(\mathrm{kpc})\end{array}$ & $\begin{array}{c}R_{200}{ }^{* *} \\
(\mathrm{kpc})\end{array}$ & RA & Dec & $z$ & $S / N$ & $\begin{array}{l}R_{500} \\
(\mathrm{kpc})\end{array}$ & $\begin{array}{l}R_{200} \\
(\mathrm{kpc})\end{array}$ & \\
\hline $\mathrm{J} 0841.4+0046$ & 130.352 & 0.777 & 0.41 & 565 & 857 & 130.351 & 0.776 & 0.44 & 4.7 & 638 & 833 & 4 \\
\hline $\mathrm{J} 1151.5+0148$ & 177.882 & 1.804 & 0.17 & 691 & 1048 & 177.917 & 1.760 & 0.16 & 4.8 & 708 & 924 & 202 \\
\hline $\mathrm{J} 1225.4+0042^{*}$ & 186.367 & 0.708 & 0.24 & 565 & 858 & 186.364 & 0.710 & 0.24 & 8.7 & 828 & 1149 & 14 \\
\hline $\mathrm{J} 1448.1-0025^{*}$ & 222.047 & -0.419 & 0.15 & - & - & 222.133 & -0.361 & 0.23 & 3.9 & 652 & 853 & 374 \\
\hline
\end{tabular}

Notes. ${ }^{(*)}$ Also in Goto et al. (2002). ${ }^{(* *)}$ Redshifts, $R_{200}$ and $R_{500}$ from Mehrtens et al. (2012): the photometric redshift for J1448.1-0025 is from Goto et al. (2002).

Table A.3. Matching between PSZ1 (Planck Collaboration XXXII 2015) and KiDS clusters.

\begin{tabular}{|c|c|c|c|c|c|c|c|c|c|c|c|c|}
\hline \multirow[b]{2}{*}{ ID } & \multicolumn{6}{|c|}{ PSZ1 } & \multicolumn{5}{|c|}{ KiDS } & \multirow[b]{2}{*}{$\begin{array}{c}\text { Sep. } \\
\left({ }^{\prime \prime}\right)\end{array}$} \\
\hline & RA & Dec & $\mathrm{S} / \mathrm{N}$ & $\begin{array}{c}\text { val. } \\
* *\end{array}$ & $z$ & $M_{\substack{5 * 0 \\
Y_{*}}}^{Y_{Z}}$ & RA & Dec & $z$ & $S / N$ & $M_{500}$ & \\
\hline G230.73+27.70* & 135.373 & -1.658 & 5.36 & 20 & 0.29 & $5.2 \pm 0.6$ & 135.393 & -1.611 & 0.33 & 13.2 & $2.9 \pm 0.5$ & 184 \\
\hline G232.76+32.70* & 140.529 & -0.441 & 4.62 & 20 & 0.32 & $4.6 \pm 0.7$ & 140.496 & -0.393 & 0.36 & 10.0 & $1.6 \pm 0.4$ & 210 \\
\hline $\mathrm{G} 286.25+62.68$ & 185.293 & 0.793 & 5.52 & 2 & - & - & 185.310 & 0.851 & 0.22 & 5.2 & $1.4 \pm 0.3$ & 219 \\
\hline
\end{tabular}

Notes. ${ }^{(*)}$ Also in Zwicky et al. (1968). ${ }^{(* *)}$ Validation status class (Planck Collaboration XXXII 2015): 2 = candidate of class $2 ; 20=$ known cluster. ${ }^{(* *)} M_{500}^{Y_{Z}}$ and $M_{500}^{\mathrm{KiDS}}$ are in units of $10^{14} M_{\odot}$. 
Appendix B: The KDR2 cluster catalog

Table B.1. An excerpt from the cluster catalog.

\begin{tabular}{|c|c|c|c|c|c|c|c|c|c|c|c|c|c|c|c|}
\hline Name & $\begin{array}{c}\text { RA } \\
\text { (deg) }\end{array}$ & $\begin{array}{c}\text { Dec } \\
(\mathrm{deg})\end{array}$ & $z$ & $S / N$ & $\begin{array}{c}\text { RA_bcg } \\
\text { (deg) }\end{array}$ & $\begin{array}{c}\text { Dec_bcg } \\
\text { (deg) }\end{array}$ & $\begin{array}{l}r \_b c g \\
\text { (mag) }\end{array}$ & $N_{500}$ & $\begin{array}{c}r_{500} \\
(\mathrm{Mpc})\end{array}$ & $\begin{array}{c}M_{500} \\
\left(10^{14} M_{\odot}\right)\end{array}$ & $\begin{array}{c}\Delta M_{500} \\
\left(10^{14} M_{\odot}\right)\end{array}$ & $N_{200}$ & $\begin{array}{c}r_{200} \\
(\mathrm{Mpc})\end{array}$ & $\begin{array}{c}M_{200} \\
\left(10^{14} M_{\odot}\right)\end{array}$ & $\begin{array}{c}\Delta M_{200} \\
\left(10^{14} M_{\odot}\right)\end{array}$ \\
\hline KDR2_J083425.9-004705 & 128.61 & -0.78 & 0.32 & 5.01 & 128.60 & -0.78 & 18.47 & 7 & 0.63 & 0.99 & 0.31 & 9 & 0.83 & 0.89 & 0.28 \\
\hline KDR2_J083613.0-002823 & 129.05 & -0.47 & 0.39 & 4.24 & 129.04 & -0.47 & 19.21 & 8 & 0.63 & 1.09 & 0.33 & 13 & 0.89 & 1.20 & 0.32 \\
\hline KDR2_J083628.6-003310 & 129.12 & -0.55 & 0.40 & 5.45 & 129.13 & -0.58 & 19.17 & 7 & 0.61 & 0.99 & 0.32 & 10 & 0.82 & 0.97 & 0.29 \\
\hline KDR2_J083706.2-001717 & 129.28 & -0.29 & 0.42 & 5.71 & 129.28 & -0.29 & 19.15 & 13 & 0.70 & 1.53 & 0.37 & 23 & 1.03 & 1.92 & 0.40 \\
\hline KDR2_J083651.8-003303 & 129.22 & -0.55 & 0.43 & 4.79 & 129.21 & -0.54 & 19.09 & 8 & 0.62 & 1.09 & 0.32 & 11 & 0.84 & 1.05 & 0.30 \\
\hline KDR2_J083704.1-002657 & 129.27 & -0.45 & 0.43 & 4.46 & 129.25 & -0.47 & 18.98 & 3 & 0.50 & 0.55 & 0.24 & 7 & 0.74 & 0.72 & 0.26 \\
\hline KDR2_J083541.8-001747 & 128.92 & -0.30 & 0.43 & 4.93 & 128.92 & -0.29 & 19.69 & 7 & 0.60 & 0.99 & 0.32 & 9 & 0.79 & 0.89 & 0.29 \\
\hline KDR2_J083414.9-002745 & 128.56 & -0.46 & 0.44 & 6.36 & 128.56 & -0.46 & 19.24 & 21 & 0.78 & 2.13 & 0.42 & 25 & 1.04 & 2.06 & 0.40 \\
\hline KDR2_J083757.4-003619 & 129.49 & -0.61 & 0.45 & 6.65 & 129.50 & -0.61 & 18.89 & 5 & 0.56 & 0.79 & 0.27 & 6 & 0.70 & 0.64 & 0.24 \\
\hline KDR2_J083555.7-001250 & 128.98 & -0.21 & 0.46 & 3.84 & 128.99 & -0.21 & 19.83 & 3 & 0.49 & 0.55 & 0.24 & 5 & 0.67 & 0.55 & 0.22 \\
\hline KDR2_J083620.2-001321 & 129.08 & -0.22 & 0.47 & 6.62 & 129.08 & -0.22 & 18.94 & 7 & 0.60 & 0.99 & 0.31 & 11 & 0.82 & 1.05 & 0.31 \\
\hline KDR2_J083433.8-001925 & 128.64 & -0.32 & 0.52 & 7.72 & 128.64 & -0.32 & 19.52 & 6 & 0.56 & 0.89 & 0.30 & 12 & 0.83 & 1.13 & 0.31 \\
\hline KDR2_J083733.4-000640 & 129.39 & -0.11 & 0.55 & 3.93 & 129.39 & -0.11 & 20.12 & 3 & 0.47 & 0.55 & 0.24 & 4 & 0.60 & 0.46 & 0.20 \\
\hline KDR2_J083748.7+000428 & 129.45 & 0.07 & 0.38 & 4.23 & 129.46 & 0.06 & 19.00 & 8 & 0.64 & 1.09 & 0.33 & 8 & 0.78 & 0.81 & 0.27 \\
\hline KDR2_J083422.8+000152 & 128.59 & 0.03 & 0.42 & 4.70 & 128.59 & 0.04 & 19.56 & 6 & 0.59 & 0.89 & 0.29 & 8 & 0.77 & 0.81 & 0.26 \\
\hline KDR2_J083410.6+004243 & 128.54 & 0.71 & 0.42 & 6.74 & 128.54 & 0.71 & 18.51 & 11 & 0.67 & 1.36 & 0.34 & 15 & 0.91 & 1.35 & 0.34 \\
\hline KDR2_J083429.3+000933 & 128.62 & 0.16 & 0.45 & 4.79 & 128.63 & 0.15 & 19.65 & 8 & 0.62 & 1.09 & 0.32 & 17 & 0.93 & 1.50 & 0.36 \\
\hline KDR2_J083414.4+002256 & 128.56 & 0.38 & 0.45 & 7.31 & 128.56 & 0.38 & 19.18 & 11 & 0.67 & 1.36 & 0.35 & 20 & 0.98 & 1.72 & 0.38 \\
\hline KDR2_J083538.4+003606 & 128.91 & 0.60 & 0.48 & 4.67 & 128.91 & 0.61 & 19.34 & 11 & 0.66 & 1.36 & 0.35 & 11 & 0.82 & 1.05 & 0.31 \\
\hline KDR2_J083510.1+001534 & 128.79 & 0.26 & 0.59 & 4.33 & 128.79 & 0.26 & 20.24 & 1 & 0.36 & 0.26 & 0.15 & 6 & 0.66 & 0.64 & 0.24 \\
\hline KDR2_J083556.2+002121 & 128.98 & 0.36 & 0.59 & 5.52 & 128.99 & 0.35 & 20.44 & 6 & 0.55 & 0.89 & 0.30 & 8 & 0.72 & 0.81 & 0.27 \\
\hline KDR2_J083723.0-013713 & 129.35 & -1.62 & 0.36 & 4.06 & 129.35 & -1.61 & 18.60 & 10 & 0.67 & 1.27 & 0.34 & 17 & 0.97 & 1.50 & 0.35 \\
\hline KDR2_J083743.4-014432 & 129.43 & -1.74 & 0.39 & 3.57 & 129.44 & -1.73 & 18.95 & 4 & 0.54 & 0.67 & 0.26 & 6 & 0.72 & 0.64 & 0.24 \\
\hline KDR2_J083747.8-020007 & 129.45 & -2.00 & 0.40 & 5.90 & 129.44 & -2.00 & 19.96 & 5 & 0.57 & 0.79 & 0.28 & 7 & 0.75 & 0.72 & 0.26 \\
\hline KDR2_J083743.2-015549 & 129.43 & -1.93 & 0.42 & 5.24 & 129.43 & -1.93 & 19.15 & 5 & 0.56 & 0.79 & 0.27 & 13 & 0.88 & 1.20 & 0.32 \\
\hline KDR2_J083555.4-013719 & 128.98 & -1.62 & 0.42 & 3.67 & 128.98 & -1.62 & 19.33 & 5 & 0.56 & 0.79 & 0.28 & 8 & 0.77 & 0.81 & 0.27 \\
\hline KDR2_J083709.4-012327 & 129.29 & -1.39 & 0.42 & 3.84 & 129.29 & -1.40 & 19.35 & 7 & 0.61 & 0.99 & 0.31 & 8 & 0.77 & 0.81 & 0.26 \\
\hline KDR2_J083726.2-015512 & 129.36 & -1.92 & 0.46 & 5.64 & 129.36 & -1.92 & 20.12 & 4 & 0.53 & 0.67 & 0.27 & 4 & 0.63 & 0.46 & 0.20 \\
\hline KDR2_J083637.7-014219 & 129.16 & -1.71 & 0.47 & 3.57 & 129.16 & -1.71 & 20.09 & 3 & 0.49 & 0.55 & 0.24 & 4 & 0.62 & 0.46 & 0.20 \\
\hline KDR2_J083705.8-014949 & 129.27 & -1.83 & 0.54 & 6.92 & 129.25 & -1.83 & 20.24 & 6 & 0.56 & 0.89 & 0.29 & 7 & 0.71 & 0.72 & 0.25 \\
\hline
\end{tabular}

Notes. The full catalog is available at the CDS. 